\title{
Comparative genome analysis of Spiroplasma melliferum IPMB4A, a honeybee-associated bacterium
}

Wen-Sui Lo ${ }^{1,2,3}$, Ling-Ling Chen ${ }^{1}$, Wan-Chia Chung ${ }^{1}$, Gail E Gasparich ${ }^{4}$ and Chih-Horng Kuo ${ }^{1,2,5^{*}}$

\begin{abstract}
Background: The genus Spiroplasma contains a group of helical, motile, and wall-less bacteria in the class Mollicutes. Similar to other members of this class, such as the animal-pathogenic Mycoplasma and the plant-pathogenic 'Candidatus Phytoplasma', all characterized Spiroplasma species were found to be associated with eukaryotic hosts. While most of the Spiroplasma species appeared to be harmless commensals of insects, a small number of species have evolved pathogenicity toward various arthropods and plants. In this study, we isolated a novel strain of honeybee-associated S. melliferum and investigated its genetic composition and evolutionary history by whole-genome shotgun sequencing and comparative analysis with other Mollicutes genomes.
\end{abstract}

Results: The whole-genome shotgun sequencing of $S$. melliferum IPMB4A produced a draft assembly that was $\sim 1.1$ $\mathrm{Mb}$ in size and covered $\sim 80 \%$ of the chromosome. Similar to other Spiroplasma genomes that have been studied to date, we found that this genome contains abundant repetitive sequences that originated from plectrovirus insertions. These phage fragments represented a major obstacle in obtaining a complete genome sequence of Spiroplasma with the current sequencing technology. Comparative analysis of S. melliferum IPMB4A with other Spiroplasma genomes revealed that these phages may have facilitated extensive genome rearrangements in these bacteria and contributed to horizontal gene transfers that led to species-specific adaptation to different eukaryotic hosts. In addition, comparison of gene content with other Mollicutes suggested that the common ancestor of the SEM (Spiroplasma, Entomoplasma, and Mycoplasma) clade may have had a relatively large genome and flexible metabolic capacity; the extremely reduced genomes of present day Mycoplasma and 'Candidatus Phytoplasma' species are likely to be the result of independent gene losses in these lineages.

Conclusions: The findings in this study highlighted the significance of phage insertions and horizontal gene transfer in the evolution of bacterial genomes and acquisition of pathogenicity. Furthermore, the inclusion of Spiroplasma in comparative analysis has improved our understanding of genome evolution in Mollicutes. Future improvements in the taxon sampling of available genome sequences in this group are required to provide further insights into the evolution of these important pathogens of humans, animals, and plants.

\section{Background}

The bacterial genus Spiroplasma contains a group of arthropod- and plant-associated endosymbionts [1-5]. Phylogenetically, the genus Spiroplasma belongs to the class Mollicutes, which are close relatives of Bacilli and other free-living Firmicutes [2,6,7]. Within Mollicutes,

\footnotetext{
* Correspondence: chk@gate.sinica.edu.tw

'Institute of Plant and Microbial Biology, Academia Sinica, Taipei, Taiwan

${ }^{2}$ Molecular and Biological Agricultural Sciences Program, Taiwan International Graduate Program, National Chung Hsing University and Academia Sinica, Taipei, Taiwan

Full list of author information is available at the end of the article
}

Spiroplasma are more closely related to the animalpathogenic genus Mycoplasma than to the plant-pathogenic 'Candidatus Phytoplasma'. However, Spiroplasma are more similar to 'Candidatus Phytoplasma' in terms of the ecological niches occupied, because both groups have complex life cycles that involve insect and plant hosts [4]. For this reason, comparative analysis of gene content among these three groups of Mollicutes can provide insights into host adaptation in these parasites [8].

While most of the Spiroplasma species that have been characterized to date appeared to be harmless commensals,

\section{Biomed Central}


some species have evolved pathogenicity toward their plant or arthropod hosts. Most of the pathogenic Spiroplasma species belong to the Citri-Chrysopicola-Mirum clade [3], notable examples include $S$. citri that causes the Citrus Stubborn Disease [9], S. kunkelii that causes the Corn Stunt Disease [10], S. phoeniceum that infects periwinkle [11], S. penaei that infects Pacific white shrimp [12,13], S. eriocheiris that infects Chinese mitten crab [14], and S. melliferum that infects honeybee [15].

To improve our understanding of these pathogenic Spiroplasma species, whole genome shotgun sequencing has been utilized as a powerful tool to investigate their metabolic capacity and possible interactions with hosts. The initial surveys of $S$. kunkelii genome revealed that this species has more genes involved in purine and amino acid biosynthesis, transcriptional regulation, cell envelope, and DNA transport than lineages from Mycoplasmataceae [16] and also a unique arrangement of genes in its ribosomal protein operon [17]. Furthermore, a comparative study within Mollicutes has identified several proteins that are shared between the phytopathogenic S. kunkelii and phytoplasma but absent in the animal-pathogenic Mycoplasma and Ureaplasma spp. [8]. More recently, draft genome sequences have been published for S. citri GII3-3X [18] and S. melliferum KC3 [19]. These more comprehensive investigations of Spiroplasma genomes revealed that their chromosomes harbor a large amount of viral sequences and also allowed for detailed characterization of their metabolic capacities. Importantly, these draft genome sequences provided a

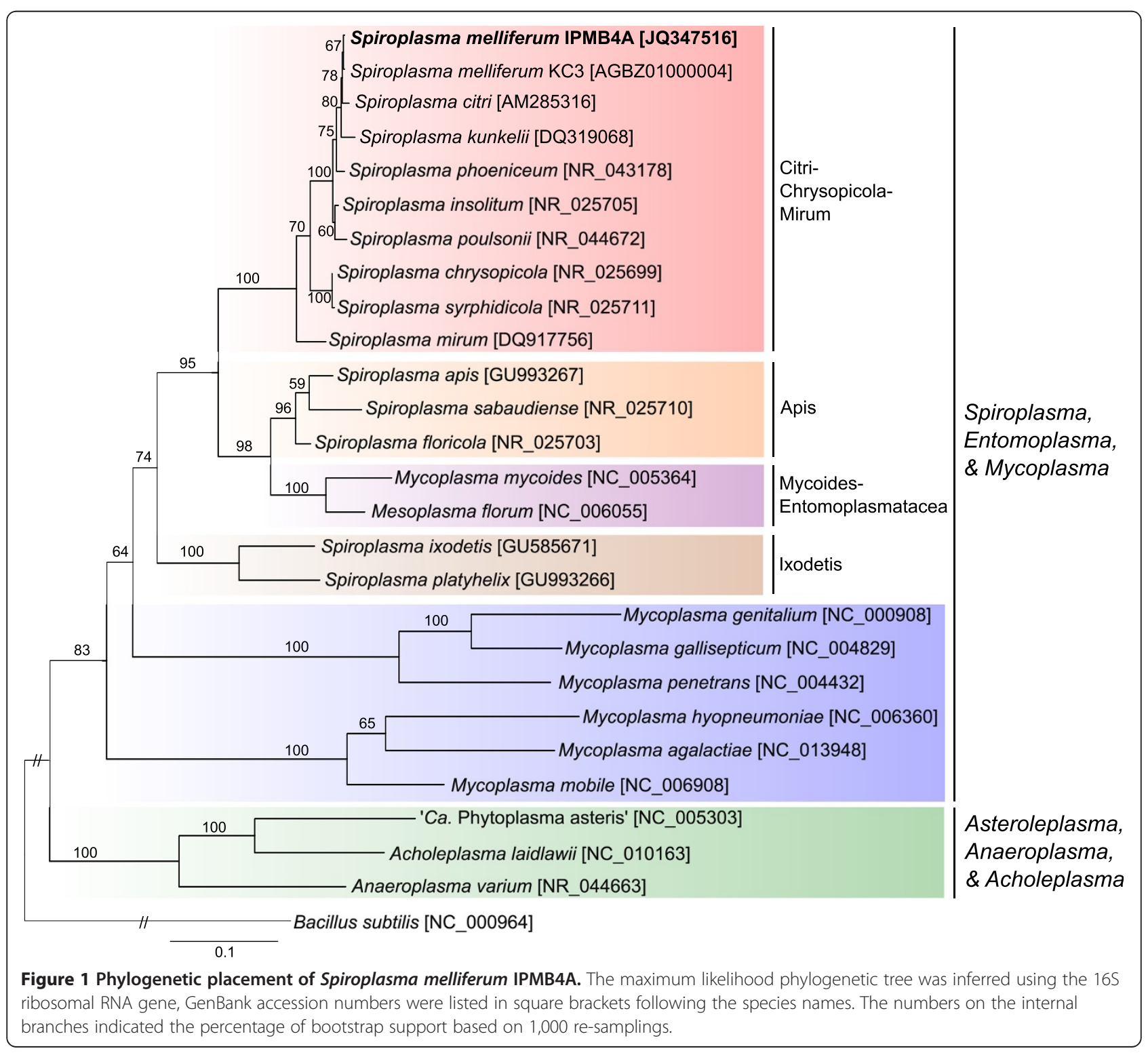


valuable resource for identification of putative virulence factors and elucidation of possible pathogenicity mechanisms $[18,19]$.

In this study, we isolated a novel strain of S. melliferum from a honeybee collected in Taiwan and investigated its genetic composition by whole-genome shotgun sequencing. Through comparative analyses with other available Mollicutes genome sequences, our aims were to characterize the genetic differences among the various lineages and to understand the evolutionary processes involved in shaping the genomes of these bacteria.

\section{Results and discussion}

\section{Species identification and phylogenetic inference}

Based on the molecular phylogeny inferred from the $16 \mathrm{~S}$ ribosomal RNA gene, the two strains of S. melliferum (IPMB4A and KC3) form a monophyletic group and S. citri is the most closely related species (Figure 1). Serological tests were confirmatory for the molecular phylogeny and supported the close association of $S$. melliferum IPMB4A with other S. melliferum strains. The deformation test showed a strong cross-reactivity between S. melliferum IPMB4A and the type strain BC$3^{\mathrm{T}}$ [15]. Positive deformation was observed up to $5,120-$ fold dilution. Against its own antisera, S. melliferum BC- $3^{\mathrm{T}}$ showed positive deformation up to 10,240 -fold dilution, but only up to 320 -fold dilution with the closely

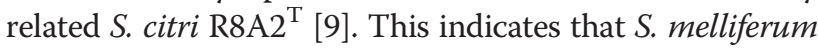
IPMB4A is a strong candidate for inclusion into Spiroplasma group I-2 with the other strains of S. melliferum.

\section{Genome assembly and annotation}

The whole genome shotgun sequencing of $S$. melliferum IPMB4A produced an assembly that contains 24 chromosomal contigs (Table 1). Consistent with the previous studies of Spiroplasma genomes [18,19], we found a large number of phage sequences in the chromosome of S. melliferum IPMB4A. Most of these phage sequences showed high similarity to plectrovirus SpV1-C74, SpV1R8A2B, and SVTS2. The presence of these long repetitive sequences was the major obstacle in obtaining a complete genome assembly. Moreover, the divergence among different copies of these repetitive plectrovirus fragments generated many single-nucleotide polymorphisms (SNPs). Most of these SNPs cannot be resolved confidently with the short 101-bp Illumina sequencing reads used in this study. To be conservative, $\sim 135 \mathrm{~kb}$ of the plectrovirusrelated regions that contained these SNPs were excluded from our final assembly. If these phage fragments were included, the assembly coverage would be similar to S. melliferum KC3 and higher than S. citri GII3-3X (Table 1). This result highlights the limitation of relying on short-read sequencing technologies for de novo assembly of highly repetitive genomes. However, the traditional
Table 1 Genome assembly statistics.

\begin{tabular}{lrrr}
\hline & $\begin{array}{r}\text { S. melliferum } \\
\text { IPMB4A }\end{array}$ & $\begin{array}{r}\text { S. melliferum } \\
\text { KC3 }\end{array}$ & $\begin{array}{r}\text { S. citri } \\
\text { Gll3-3X }\end{array}$ \\
\hline $\begin{array}{l}\text { Number of chromosomal } \\
\text { contigs }\end{array}$ & 24 & 4 & 39 \\
$\begin{array}{l}\text { Combined size of } \\
\text { chromosomal contigs (bp) }\end{array}$ & $1,098,846$ & $1,260,174$ & $1,525,756$ \\
$\begin{array}{l}\text { Estimated chromosomal } \\
\text { size (bp) }\end{array}$ & $1,380,000$ & $1,430,000$ & $1,820,000$ \\
Estimated coverage (\%) & & & \\
G+C content (\%) & 79.6 & 88.1 & 83.8 \\
Coding density (\%) & 27.5 & 27.0 & 25.9 \\
Protein-coding genes & 85.1 & 83.0 & 80.2 \\
Length distribution & 932 & 1,222 & 1,905 \\
(Q1/Q2/Q3) (a.a.) & $176 / 280 / 440$ & $119 / 233 / 376$ & $83 / 149 /$ \\
Plectrovirus proteins & & & 286 \\
Hypothetical proteins & 11 & 132 & 375 \\
Annotated pseudogenes ${ }^{1}$ & 337 & 485 & 519 \\
rRNA operon & 12 & 12 & 401 \\
tRNA & 1 & 1 & 1 \\
Number of plasmids & 32 & 31 & 32 \\
\hline
\end{tabular}

${ }^{1}$ For S. melliferum IPMB4A, putative pseudogenes were annotated with the'/ pseudo' tag in gene feature as suggested by the NCBI GenBank guidelines and were not counted in the total number of protein-coding genes. For $S$. melliferum KC3 and S. citri GIII-3X, putative pseudogenes were annotated by adding the term 'truncated' in the CDS product description field and were included in the total number of protein-coding genes.

approach of using clone libraries for Sanger shotgun sequencing has a much higher cost and may not be a good choice if the main focus is on the unique regions of the genome.

The exclusion of phage fragments reduced the number of annotated plectrovirus proteins in the $S$. melliferum IPMB4A assembly compared to the closely related S. melliferum KC3 (Table 1). With the elimination of these short plectrovirus proteins and other potential falsepositive gene predictions (see Methods) from the annotation, we observed a shift in the length distribution of the annotated protein-coding genes compared to the other two Spiroplasma genomes (Table 1). Nonetheless, our conservative approach in assembly and annotation mainly removed viral proteins and short hypothetical proteins, thus was not expected to introduce biases in the downstream metabolic analysis. Intriguingly, in contrast to the abundance of plasmids in S. melliferum KC3 [19] and S. citri GII3-3X $[18,20]$, we did not recover any plasmid-like contig in our draft assembly of S. melliferum IPMB4A genome (Table 1). This lack of plasmid-like contig is consistent with the negative result from our plasmid extraction tests. This finding may reflect the true absence of plasmids in this particular strain. Alternatively, it is possible that the plasmid(s) had been lost during the process of initial isolation or subsequent culturing in the laboratory. 
Among the 932 annotated protein-coding genes in the S. melliferum IPMB4A genome, 392 have specific functional assignments according to the COG categories (Figure 2). Genes involved in translation and ribosomal structure (COG category J) represented the most abundant functional category, mostly due to the large number of ribosomal proteins and tRNA synthetases found in the genome. The second most abundant category contained genes involved in replication, recombination, and repair (COG category $\mathrm{L}$ ). In addition to the DNA replication machinery such as DNA polymerases (e.g., dnaE, dnaN, dnaX, holA, holB, and polC) and other related proteins (e.g., dnaA, dnaB, dnaG, ligA, polA, ssbA, etc.), we also found genes involved in nucleotide excision repair (e.g., $u v r A, u v r B$, and $u v r C$ ) and several DNA topoisomerases (e.g., gyrA, gyrB, parC, and parE). However, the machineries for mismatch repair (e.g., mutS, mutL, mutH, exoI, exoX, recJ, etc.) and homologous recombination (e.g., $\operatorname{rec} A, \operatorname{rec} B, \operatorname{rec} C$, etc.) appeared to be missing.

\section{Comparative analysis with S. melliferum KC3 and S. citri} The alignment among the three draft Spiroplasma genomes revealed extensive rearrangements between the closely related $S$. melliferum and $S$. citri (Figure 3A). This low level of conservation in synteny was unexpected for several reasons. First, the average nucleotide sequence identity between these two species was $\sim 99 \%$, suggesting that the divergence time was quite short [21]. For comparison, two other groups of related bacteria with similar (i.e., between-strain comparison in Mycoplasma hyopneumoniae; see Figure 3B) or even higher (i.e., between-species comparison in Bacillus; see Figure 3C) levels of divergence both had a higher level of conservation in their chromosomal organization. Second, in a previous study that compared the physical maps of S. melliferum and S. citri, the authors found that the overall organization was similar between these two species [22]. However, this discrepancy may be due to the higher resolution in detecting genome rearrangements provided by genome sequencing compared to pulsed-field gel electrophoresis (PFGE). Finally, these two species lack several genes that were involved in homologous recombination. For example, the recombinase A (encoded by $\operatorname{rec} A$ ) was reduced to 231 a.a. in $S$. melliferum by a premature stop codon and 130 a.a. in $S$. citri by one or more truncations of its coding region [18,23]; for comparison, the full length RecA protein in Mycoplasma mycoides contains 345 a.a. (GenBank accession number NP_975407). Although these Spiroplasma genomes contain a large number of direct and inverted repeats originated from the invasion of plectroviruses, the pesudogenization of $r e c A$ was expected to promote genome stability [24,25]. One possible explanation of this paradox is that these extensive genome rearrangements were facilitated by the large number of repetitive sequences [26] and had occurred soon after the species divergence, while the independent losses of homologous recombination in these two species were relatively recent events. More extensive taxon sampling of

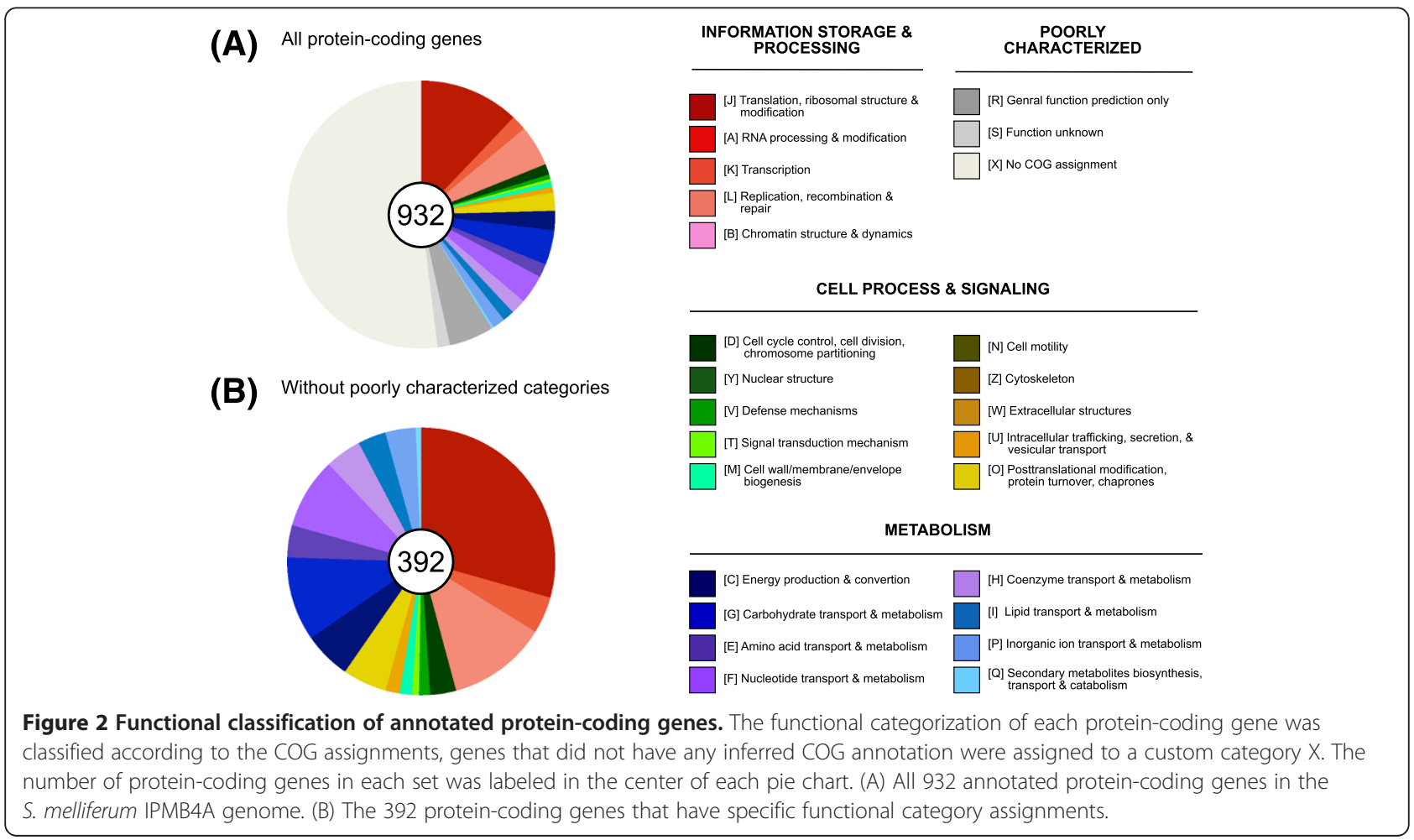




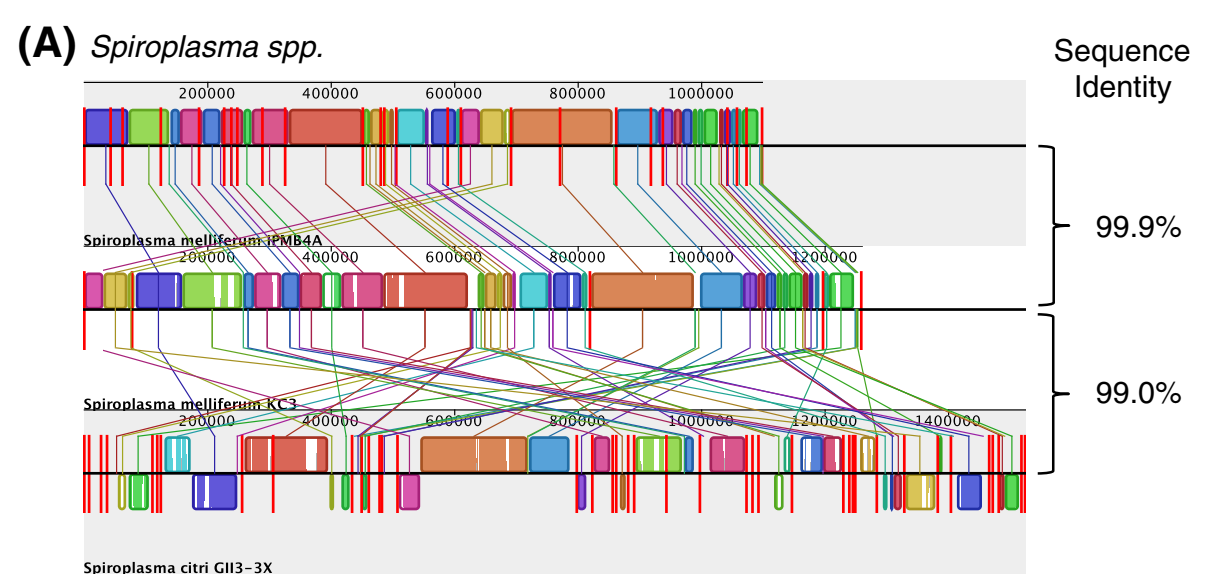

(B) Mycoplasma hyopneumoniae

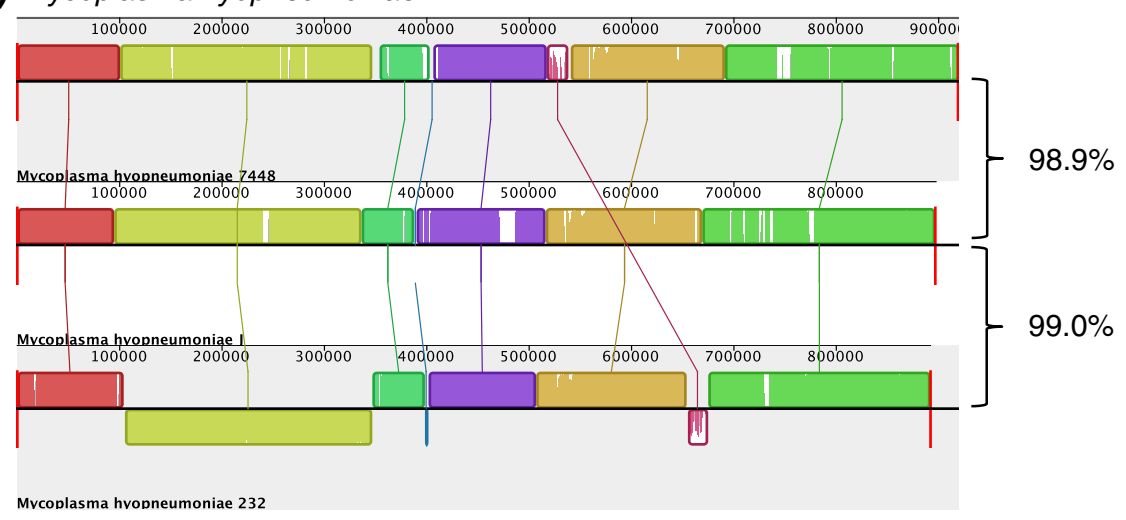

(C) Bacillus spp.

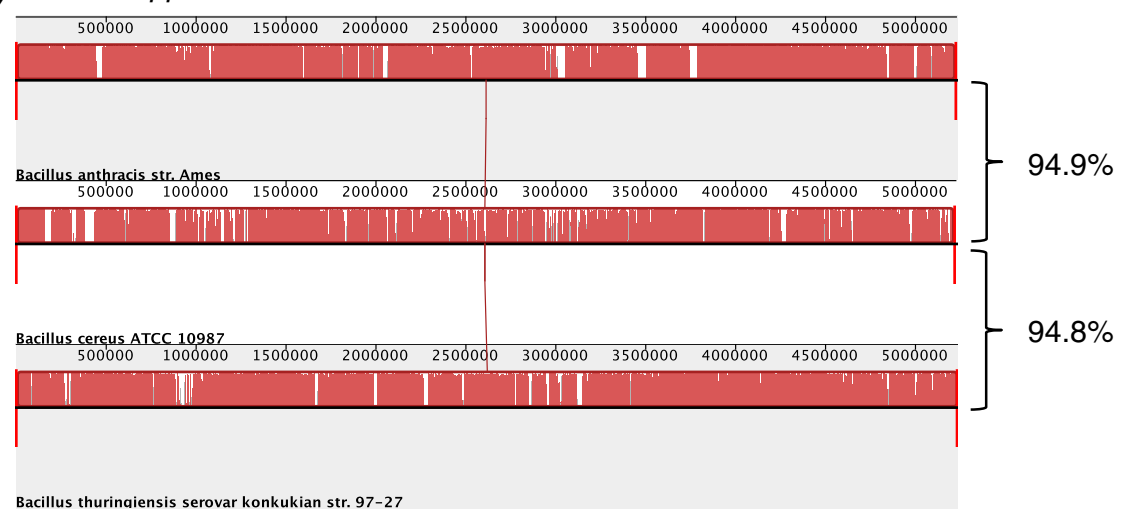

Figure 3 Extensive level of genome rearrangement between closely related Spiroplasma spp. The color blocks represent regions of homologous backbone sequences without rearrangement among the genomes compared. The vertical red bars indicate the boundaries of individual contigs. The average nucleotide sequence identities were calculated based on single-copy genes that were conserved among the three genomes compared in each group. (A) Comparison among Spiroplasma melliferum IPMB4A, S. melliferum KC3, and S. citri GII3-3X. (B) Comparison among Mycoplasma hyopneumoniae strains 7448, J, and 232. (C) Comparison among Bacillus anthracis str. Ames, B. cereus ATCC 10987, and B. thuringiensis serovar konkukian str. 97-27. 
Spiroplasma genomes, including additional strains from these two species and appropriate outgroup species, are required to investigate this hypothesis.

To compare the gene content between S. melliferum and $S$. citri, we identified the homologous genes among the three available genomes from these two species and classified these genes based on their patterns of presence and absence (Figure 4A and Additional file 1). Examination of these results can improve our understanding of the genetic differentiation between these two species. However, two caveats exist due to the nature of these data sets. First, all three Spiroplasma genomes used in this comparison were incomplete draft sequences, such that the absence of genes from any particular genome cannot be confirmed. Second, due to the lack of an appropriate outgroup (e.g., an additional Spiroplasma species in the Citri clade), we cannot establish the directionality of evolutionary changes based on the patterns of presence and absence. When the ongoing genome sequencing of $S$. kunkelii $[8,16,17]$ reaches completion in the future, combined analysis using a phylogenetic framework will provide more insights into the gene content evolution of these pathogenic Spiroplasma species.

Despite these potential shortcomings, inspection of the gene lists suggested that this comparison of gene content provided results that were consistent with our expectations. We found that both S. melliferum KC3 and $S$. citri GII3-3X contained large numbers of genomespecific genes and also shared 85 homologous gene clusters that were absent in S. melliferum IPMB4A. The majority of these genes encoded hypothetical proteins and proteins of viral origin. These observations were expected due to our exclusion of most plectrovirus fragments from the assembly of $S$. melliferum IPMB4A genome (see above). Furthermore, examination of these gene lists provided insights into the biology of these bacteria. Both species have phosphotransferase system (PTS) transporters and enzymes for the uptake and utilization of trehalose, glucose, and fructose (i.e., treB, ptsG, fruA, and crr; see Figure 5A and Additional file 1). These findings were consistent with our current knowledge of spiroplasma biology. For example, trehalose exists in higher concentration than glucose or fructose in insect haemolymph [27] and may be an important carbon source for spiroplasmas during their life stage in insect vectors. In addition, both species were known to utilize glucose and fructose $[9,15]$ and the preferential uptake of fructose by $S$. citri is linked to its phytopathogenicity $[28,29]$.

Intriguingly, compared to the plant-pathogenic S. citri, the insect-pathogenic $S$. melliferum has additional genes involved in the utilization of cellobiose, chitin, and $N$ acetylmuramic acid (MurNAc) (i.e., celA, celB, chiA, $n a g E$, and murP; see Figure $5 \mathrm{~B}$ and Additional file 1). The presence of these genes may be linked to its adaptation to the honeybee host. For example, the ability to degrade chitin can facilitate its invasion of the host tissues (e.g., through the chitinous peritrophic matrix, gut epithelium, or exoskeleton, etc.) and the ability to utilize cellobiose may enhance its fitness by allowing access to the partially hydrolyzed pollen cell walls in honeybee

\section{(A)}

\section{Spiroplasma melliferum} IPMB4A

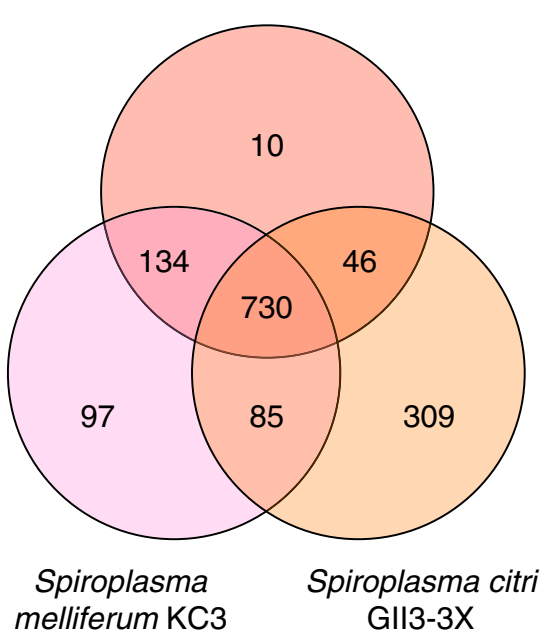

(B) Spiroplasma melliferum IPMB4A

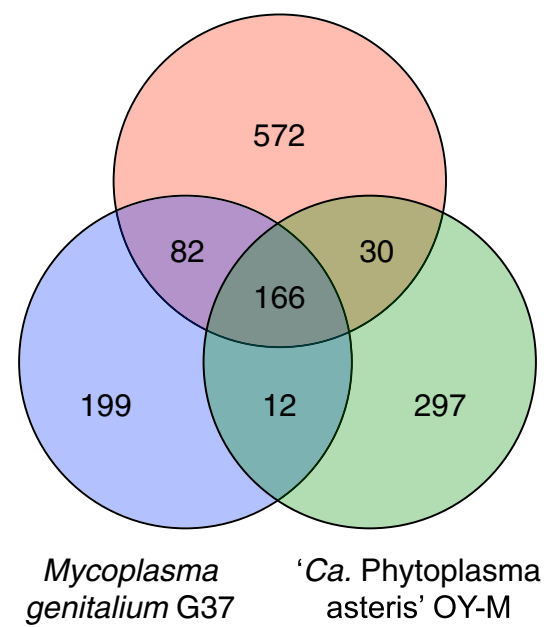

Figure 4 Numbers of shared and genome-specific homologous gene clusters. The Venn diagrams show the number of shared and genome-specific homologous gene clusters among the genomes compared. (A) Comparison among Spiroplasma melliferum IPMB4A, S. melliferum KC3, and S. citri GII3-3X. (B) Comparison among S. melliferum IPMB4A, Mycoplasma genitalium G37, and 'Candidatus Phytoplasma asteris' OY-M. 


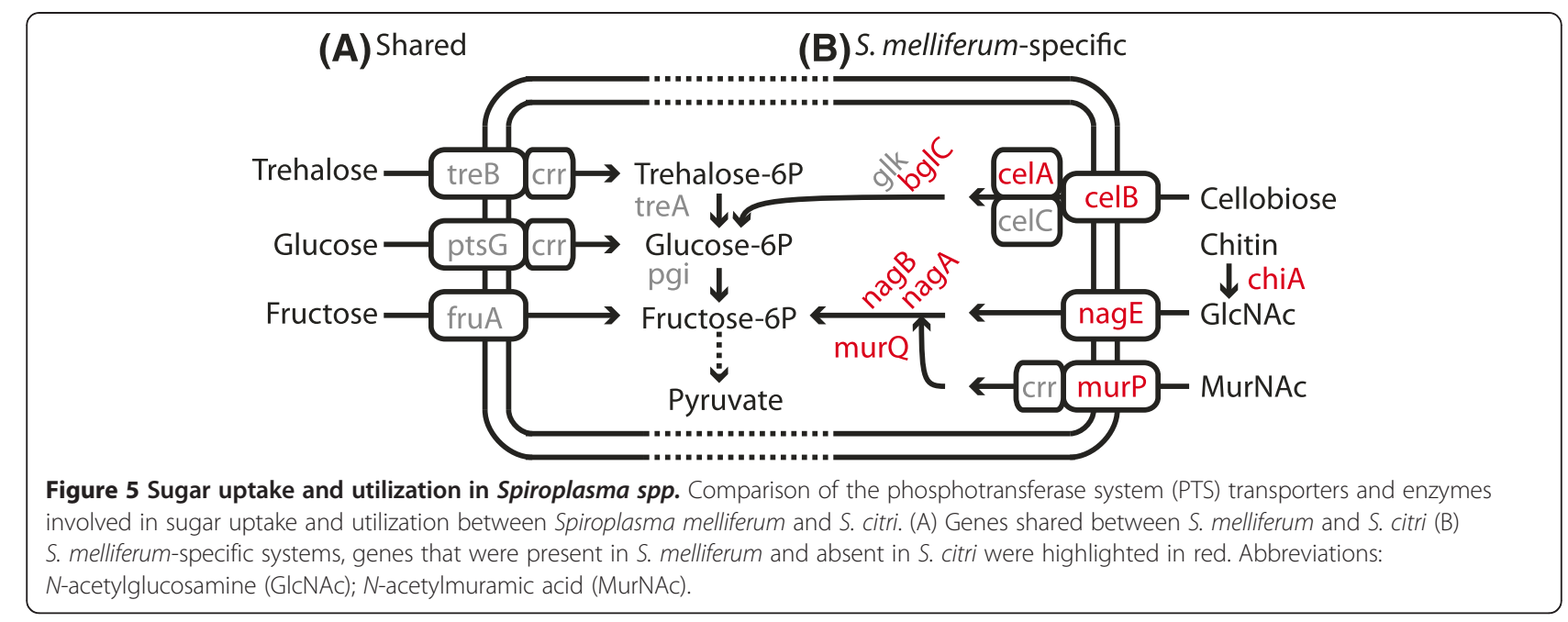

diet for additional carbon sources. More interestingly, several of these S. melliferum-specific genes (e.g., chiA, celA, and $c e l B$ ) were adjacent to plectrovirus fragments on the chromosome, suggesting that these genes may have been acquired through phage-mediated horizontal gene transfers. The sequence similarity search against the GenBank database revealed that the chitinase in S. melliferum has no putative homolog within the class Mollicutes and the most similar genes were found in the order Lactobacillales, including the genera Lactococcus and Enterococcus.
Comparative analysis with mycoplasma and phytoplasma For comparative genomics analysis at a deeper divergence level (Figure 1), we compared the gene content of $S$. melliferum IPMB4A with two other representative Mollicutes species: Mycoplasma genitalium G37 (GenBank accession number NC_000908) and 'Candidatus Phytoplasma asteris' OY-M (NC_005303). The inclusion of the M. genitalium, which has the smallest genome among sequenced Mollicutes, is useful for defining the most conserved core gene set in these bacteria. Among these three genomes, S. melliferum IPMB4A is the largest
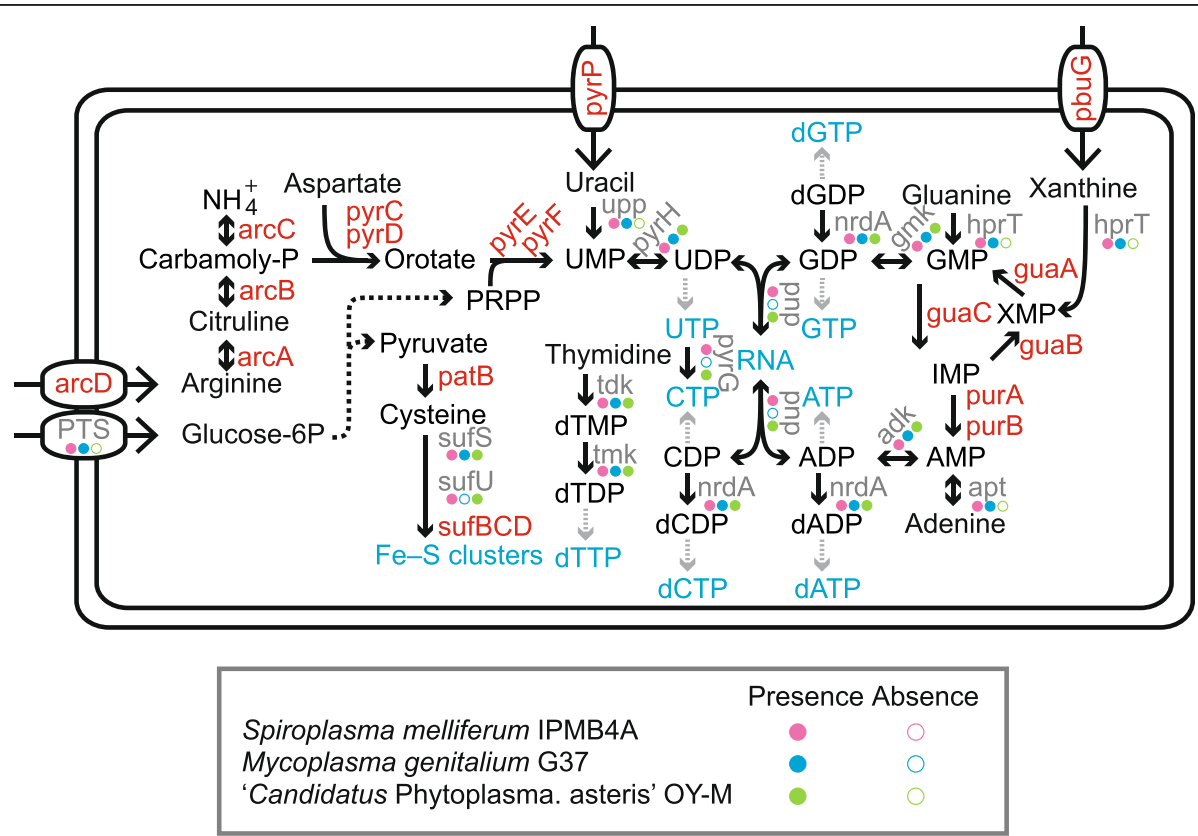

Figure 6 Highlights of selected metabolic pathways in Mollicutes. The analysis was based on a three-way comparison among Spiroplasma melliferum IPMB4A, Mycoplasma genitalium G37, and 'Candidatus Phytoplasma asteris' OY-M. The color-coded circles indicated the presence (filled) or the absence (unfilled) of a gene in each genome. Genes that were present in S. melliferum IPMB4A and absent in the other two genomes were highlighted in red. 
one and contains the highest number of genome-specific genes (Figure $4 \mathrm{~B}$ and Additional file 2). Examination of these genes revealed that Spiroplasma possesses more flexible metabolisms and consequently a lower degree of host dependence. In the initial isolation and characterization, S. melliferum was reported to ferment glucose and to hydrolyze arginine [15]. Our pathway analysis revealed that these two features may be important in its physiology. In addition to the PTS transporter for glucose uptake, $S$. melliferum possesses all genes required for glycolysis to convert glucose-6-phosphate into pyruvate (Figure 5 and Figure 6). Pyruvate may be used for the production of cysteine by an aminotransferase (patB) and the biosynthesis of Fe-S clusters by other downstream genes (i.e., sufS, sufU, sufB, sufC, and sufD; see Figure 6). Alternatively, pyruvate may also be converted into acetyle-CoA by the pyruvate dehydrogenase complex (i.e., $p d h A, p d h B$, and $p d h C)$. Other than glycolysis, S. melliferum also has the genes in the pentose phosphate pathway (e.g., pgi, tkt, rpe, rpiB, and prs) for producing 5-phosphoribosyl diphosphate (PRPP), which is a precursor for pyrimidine metabolism (Figure 6).

The ability to uptake and metabolize arginine provides $S$. melliferum with a source of nitrogen and also precursors for de novo nucleotide biosynthesis. The possession of the entire gene set (i.e., $\operatorname{arc} A, \operatorname{arc} B, \operatorname{arc} C, \operatorname{pyr} C, \operatorname{pyr} D$, pyrE, and pyrF; see Figure 6) for converting arginine to uridine monophosphate (UMP) is rare among Mollicutes. Other than S. melliferum, we only found that Mycoplasma penetrans [30] contains all these genes as well. However, result from database searches demonstrated sporadic distribution patterns of individual genes among different Mycoplasma species (e.g., some of these genes were found in $M$. capricolum, M. hominis, M. putrefaciens, etc.). In addition to the de novo biosynthesis of UMP, S. melliferum may acquire xanthine and uracil by permeases (e.g., pbuG and pyrP). These nucleobase transporters were also rare among Mollicutes. Other than S. melliferum, these permeases were only found in S. citri and Mesoplasma florm. Although these findings may indicate independent gene acquisitions by horizontal gene transfers, a more plausible explanation is that the common ancestor of the SEM (Spiroplasma, Entomoplasma, and Mycoplasma) clade may have maintained these genes for nucleotide transport and metabolism following its divergence from the AAA (Asteroleplasma, Anaeroplasma, and Acholeplasma; including phytoplasmas) clade. Subsequently, the nutrient-rich environments provided by the eukaryotic hosts reduced the selective pressure for maintaining these genes in the genome and allowed parallel gene losses in most SEM lineages. In addition to the genes discussed above, we also found several Spiroplasma-specific genes that are associated with its unique biology among Mollicutes. For example,
S. melliferum IPMB4A has five copies of $m r e B$ (encoding for the cell shape determining protein MreB) and two copies of spiralin (which are likely to be involved in its interactions with the insect host [31]). Furthermore, S. melliferum IPMB4A has several genes involved in lipid metabolism, such as the non-mevalonate pathway for terpenoid backbone biosynthesis (i.e., $d x s, d x r$, ispD, ispF, ispG, and ispH; see Additional file 2).

Among the three genomes included in this comparative analysis, $M$. genitalium contained the fewest number of protein-coding genes (i.e., 475 compared to 749 in 'Candidatus Phytoplasma asteris' and 932 in S. melliferum). Given these numbers, one might expect that $S$. melliferum would shared more genes with 'Candidatus Phytoplasma asteris' than with M. genitalium. However, the homologous gene cluster identification showed that $S$. melliferum and M. genitalium actually shared more genes to the exclusion of 'Candidatus Phytoplasma asteris' (Figure 4B and Additional file 2). This finding is consistent with the close evolutionary relationship between Spiroplasma and Mycoplasma. Many of the genes in this list represented gene losses in the phytoplasma lineage. For example, the absence of $\mathrm{F}_{0} \mathrm{~F}_{1}$-type ATP synthase (e.g., $\operatorname{atp} A, \operatorname{atp} B, \operatorname{atpD}$, $\operatorname{atpF}, \operatorname{atp} F$, atpG, and $a t p H)$ and pentose phosphate pathway (e.g., tkt, rpiB, prs, deoC) were noted in the initial genome sequencing of phytoplasmas [32,33]. Furthermore, the phytoplasma-specific losses of purine salvage pathway (apt and $h p r T)$, pyrimidine metabolism $(\operatorname{tr} x B)$, formylation of methionyl-tRNA ( $f m t$ and $f o l D$ ), and potassium ion uptake ( $k \operatorname{tr} A$ and $k \operatorname{tr} B$ ) were noted in our previous large-scale comparative analysis between mycoplasmas and phytoplasmas [34].

According to the phylogenetic relationship among these three lineages, the genes that are shared between S. melliferum and 'Candidatus Phytoplasma asteris' but absent in the M. genitalium genome would be of particular interest (Additional file 2). Although some of these genes may reflect accelerated sequence divergence or gene losses in $M$. genitalium, others may provide insights into the adaptation of spiroplasmas and phytoplasmas to their similar ecological niches. Examination of the 30 homologous gene clusters identified in this study mostly confirmed the results of a previous study that investigated this topic [8] and also provide additional candidate genes for future characterization.

In the initial survey of an $85-\mathrm{kb}$ segment of the $S$. kunkelii CR2-3X genome, it was reported to have a unique arrangement of ribosomal protein genes [17]. Compared to M. capricolum, Bacillus subtilis, and Escherichia coli, the intergenic regions are much shorter in S. kunkelii. When we compared this region across the available Mollicutes genomes, we found that the compactness of this operon is not necessarily a defining feature of Spiroplasma genomes. For example, the highly reduced $M$. 
genitalium genome has similar or even shorter intergenic regions between neighboring genes. However, we noted that the three Spiroplasma species examined (i.e., $S$. kunkelii, S. citri, and S. melliferum) all have the same set of three genes (i.e., secY, adk, and infA) located downstream of ribosomal protein L15 ( $r p l O)$. In contrast, several phytoplasma and Mycoplasma species, including M. mycoides that is nested within the Spiroplasma clade (Figure 1), all have an additional methionine aminopeptidase (map) located between $a d k$ and infA. As the taxon sampling of available Spiroplasma genomes continues to improve, it will be interesting to investigate if this relocation of the map gene is specific to the Citri clade or shared by other Spiroplasma lineages.

\section{Conclusions}

The genome survey by shotgun sequencing revealed that several Spiroplasma species harbor abundant plectrovirus fragments in their genome. Because of the repetitive nature of these viral sequences and the limitations of current sequencing technologies, obtaining the complete genome sequence of these bacteria has remained extremely challenging. Nonetheless, our analysis of the partial genome sequences of $S$. melliferum and $S$. citri provided glimpses into the genome architecture and metabolic capacity of these bacteria. Furthermore, comparative analysis with other Mollicutes demonstrated that the availability of Spiroplasma genome sequences is of fundamental importance in improving our knowledge of the evolutionary history in this class. Through comparison with the animal-pathogenic Mycoplasma and the plant-pathogenic 'Candidatus Phytoplasma', results from this study suggested that the extensive gene losses observed in these bacteria may have occurred independently among different lineages, rather than mainly in their common ancestor as previously thought [34]. For future study, additional taxon sampling of Spiroplasma genomes would further improve the reconstruction of ancestral states and the inference of evolutionary history in these important pathogenic bacteria.

\section{Methods}

\section{Strain isolation and DNA preparation}

The bacterial strain S. melliferum IPMB4A was isolated from a honeybee collected at the main campus of the National Taiwan University (Taipei, Taiwan) on April 13, 2011 following the protocol described in a previous study [35]. Briefly, each honeybee was homogenized in 2 $\mathrm{ml}$ of the $\mathrm{R}_{2}$ medium [36] using a ceramic mortar and pestle set. The homogenized samples were allowed to sit for 20 minutes before passing through a 0.45 um filter (Millipore, USA) by gravity without added pressure. The filtered medium was diluted $20 \mathrm{X}$ and incubated at $31^{\circ} \mathrm{C}$ without shaking. The culture tubes were checked daily for color change, samples that turned yellow were checked using dark field microscopy to confirm the presence of helical-shaped spiroplasma cells and triply cloned by colony isolation on $1.5 \%$ agar plates. The pathogenicity of the IPMB4A strain was unknown. For PCR and whole-genome shotgun Illumina sequencing (see below), we isolated the genomic DNA of S. melliferum IPMB4A using the Wizard Genomic DNA Purification Kit (Promega, USA) according to the manufacturer's protocol.

\section{Pulsed-field gel electrophoresis}

To determine the genome size of S. melliferum IPMB4A, we performed pulsed-field gel electrophoresis (PFGE) based on established protocols [37,38]. Briefly, $40 \mathrm{ml}$ of saturated cell culture was obtained by incubation at $30^{\circ} \mathrm{C}$ without shaking for 72 hours. Cells were collected by centrifugation at 12,000 $\mathrm{xg}$ for 35 minutes. The resulting pellet was gently re-suspended in 400 ul of TSE buffer (20 mM Tris/Cl, 10\% sucrose, $0.05 \mathrm{M}$ EDTA, pH 8.0) and warmed to $30^{\circ} \mathrm{C}$ before mixing with an equal volume of pre-melted 2\% low melting temperature agarose (Lonza, Switzerland). The mixture was quickly transferred into a cold gel plug mold and allowed to solidify at $4^{\circ} \mathrm{C}$ for 30 minutes. The gel plugs were incubated in lysis buffer $(0.5$ M EDTA, $1 \%$ N-lauroylsarcosine, $\mathrm{pH} 8.0,1 \mathrm{mg} / \mathrm{ml}$ proteinase $\mathrm{K}$ added freshly) at $55^{\circ} \mathrm{C}$ for three days with three buffer changes daily. The PFGE step was carried out using a CHEF Mapper XA System (Bio-Rad, USA) under the following conditions: 24-hour run, $120^{\circ}$ included angle, 24.03-120 sec switch-time ramp, $6 \mathrm{~V} / \mathrm{cm}, 0.5 \mathrm{X} \mathrm{TBE}$ (0.045 M Tris/borate, $1 \mathrm{mM}$ EDTA), $14^{\circ} \mathrm{C}, 1.0 \%$ pulsedfield certified agarose (Bio-Rad, USA). The Saccharomyces cerevisiae chromosomal DNA (Bio-Rad, USA) was used as the size standards to estimate the molecular weight of the S. melliferum IPMB4A chromosome.

\section{Molecular phylogenetic inference}

To perform molecular phylogenetic inference, the partial $16 \mathrm{~S}$ ribosomal RNA gene was amplified by using the primer pair 8F (5'-agagtttgatcctggctcag-3') and 1492R (5'-ggttaccttgttacgactt-3'). Each 50 ul reaction mix contained $1 \mathrm{ul}$ of PfuUltra Fusion HotStart DNA polymerase (Agilent, USA), $5 \mathrm{ul}$ of the supplied 10X buffer, $2 \mathrm{mM}$ $\mathrm{MgCl}_{2}, 0.2 \mathrm{uM}$ of each primer, $250 \mathrm{uM}$ of each dNTP, and $100 \mathrm{ng}$ of DNA template. The cycling conditions were: (1) an initial activation step at $95^{\circ} \mathrm{C}$ for $3 \mathrm{~min}$; (2) 25 cycles of $95^{\circ} \mathrm{C}$ for $40 \mathrm{sec}, 55^{\circ} \mathrm{C}$ for $40 \mathrm{sec}$, and $72^{\circ} \mathrm{C}$ for $40 \mathrm{sec}$; and (3) a final extension step for $5 \mathrm{~min}$. The PCR product of expected size was isolated using agarose gel electrophoresis and purified using QIAquick Gel Extraction Kit (Qiagen, Germany) according to the manufacturer's protocol. The purified PCR product was cloned using the Zero Blunt TOPO PCR Cloning Kit 
(Invitrogen, USA) and sequenced using the BigDye Terminator v3.1 Cycle Sequencing Kit on an ABI Prism 3700 Genetic Analyzer (Applied Biosystems, USA). The consensus sequence from 11 individual clones (1,442-bp, excluding the PCR primers) was used as the representative sequence and deposited in NCBI GenBank under the accession number JQ347516.

This partial 16S rRNA gene sequence was used as the query to run BLASTN $[39,40]$ against the NCBI nt db [41] to identify homologous sequences from related species. These sequences (see Figure 1 for accession numbers) were aligned using MUSCLE [42] with the default settings. The resulting multiple sequence alignment was used to infer the species phylogeny using the maximum likelihood program PhyML [43]. The transition/transversion ratio, proportion of invariable sites, and the gamma distribution parameter (with four categories of substitution rates) were estimated from the alignment in the maximum likelihood framework. To estimate the level of support for each internal branch, we generated 1,000 non-parametric bootstrap samples of the alignment by using the SEQBOOT program in the PHYLIP package [44] and repeated the phylogenetic inference as described above.

\section{Serology test}

The deformation test was performed using S. melliferum IPMB4A against the hyperimmune antisera of S. melliferum $\mathrm{BC}-3^{\mathrm{T}}$ (ATCC 33219) as described previously [45]. Positive reactions were indicated by the visual appearance of grapelike clustering and clumping of the spiroplasma cells, indicative of a reaction between the cell membrane and the antisera. The appearance of normal helical-shaped spiroplasmas indicated no reaction to the antisera. A dilution is designated as positive when over $50 \%$ of the spiroplasma cells present show a positive reaction. The test was performed three times.

\section{Whole-genome shotgun sequencing}

To obtain the genome sequence of $S$. melliferum IPMB4A, we used a commercial sequencing provider (Yourgene Bioscience, Taipei, Taiwan) for whole-genome shotgun sequencing based on the Illumina HiSeq 2000 platform (Illumina, USA). Two separate libraries were prepared and sequenced, including one paired-end library (insert size $=365 \mathrm{bp}, 79,924,629$ read-pairs, approximately $16 \mathrm{~Gb}$ of raw data) and one mate-pair library (insert size $=\sim 4.5 \mathrm{~kb}, 20,463,824$ read-pairs, approximately $4 \mathrm{~Gb}$ of raw data).

\section{Genome assembly and annotation}

The paired-end Illumina reads were used for de novo genome assembly by the program Velvet-SC [46]. The raw reads were trimmed from the 5 -end at the first position that has a quality score of lower than 20 , all reads that were shorter than $70 \mathrm{bp}$ after this quality trimming step were discarded. This resulted in 14,129,347 unpaired and $80,693,442$ paired reads. The k-mer, expected coverage, and coverage cutoff were set to $79,4,000$, and 100 , respectively.

The 25 contigs that were longer than $1 \mathrm{~kb}$ after this initial assembly step were used as the starting point for our iterative assembly correction process. For each iteration, we mapped all raw reads (including paired-end and matepair reads) to the assembled contigs using BWA [47] and visualized the results using IGV [48]. Neighboring contigs with mate-pair support for continuity were merged into scaffolds and polymorphic sites were examined using the MPILEUP program in the SAMTOOLS package [49]. For regions that appear to have been mis-assembled (often associated with aberrant coverage patterns), we extracted the raw reads that were mapped to the corresponding regions and re-assemble these regions by PHRAP [50]. These re-assembled regions were incorporated into the assembly and verified in the next iteration. We repeated this iterative process until no improvement could be made with the method described.

At the final step of our assembly process, we removed regions that contained unresolvable polymorphisms (all corresponded to repetitive plectrovirus fragments) and un-filled gaps from the scaffolds. The resulting contigs were processed using RNAmmer [51], tRNAscan-SE [52], and PRODIGAL [53] for gene prediction. The gene name and description for the protein-coding genes were assigned based on the orthologous genes identified by OrthoMCL [54] in other Mollicutes genomes and BLASTP [39,40] searches against NCBI nr db [41]. For functional categorization, all protein sequences were annotated by utilizing the KAAS tool [55] provided by the KEGG database [56,57] using the bi-directional best hit method. In addition to the default Prokaryotes representative set, we included selected Tenericutes and Firmicutes in the reference genomes. The complete list of abbreviated genome ids selected includes: hsa, dme, ath, sce, pfa, eco, sty, hin, pae, nme, hpy, rpr, mlo, bsu, sau, lla, spn, cac, mge, mtu, ctr, bbu, syn, aae, mja, afu, pho, ape, mpn, mmy, mmo, maa, uur, poy, acl, mfl, bha, bce, lca, and cbo. The KEGG orthology assignments were further mapped to the COG functional category assignment $[58,59]$ to generate summary statistics (Figure 2). Genes that did not have any COG functional category assignment were assigned to a custom category (category X: no COG assignment). Finally, the annotation was manually curated to incorporate evidence from each of the approaches described above and to ensure the consistency of gene descriptions.

To correct for possible over-annotation, predicted protein-coding genes that were shorter than 100 amino acids, had low confidence scores as inferred by 
PRODIGAL [53], and lacked any functional predictions or putative homologous sequences were eliminated from the final annotation. To correct for possible under-annotation, genes that were conserved between the closely related S. melliferum KC3 and S. citri GII3-3X genomes but were absent in our initial gene prediction were used as queries to search against the contigs.

This Whole Genome Shotgun project has been deposited at DDBJ/EMBL/GenBank under the accession AMGI00000000 (BioProject PRJNA80357). The version described in this paper is the first version, AMGI01000000.

\section{Comparative analysis with other genomes}

To examine the extent of genome rearrangement in Spiroplasma and other related bacteria, we used MAUVE [60] to compare S. melliferum IPMB4A and the following genomes (see Figure 3): S. melliferum KC3 (GenBank accession numbers AGBZ01000001-AGBZ01000004), S. citri GII3-3X (AM285301-AM285339), Mycoplasma hyopneumoniae 7448 (NC_007332), M. hyopneumoniae J (NC_007295), M. hyopneumoniae 232 (NC_006360), Bacillus anthracis str. Ames (NC_003997), B. cereus ATCC 10987 (NC_003909), and B. thuringiensis serovar konkukian str. 97-27 (NC_005957). The average sequence identities between genomes were calculated by the DNADIST program in the PHYLIP package [44] using conserved singlecopy genes identified by OrthoMCL [54] within each group.

To identify lists of shared and genome-specific genes at different levels of evolutionary divergence, we compared the gene content of S. melliferum IPMB4A with other representative Mollicutes genomes (Figure 4). Because of the varying levels of sequence divergence, the sequence similarity search step in the OrthoMCL [54] analysis was conducted at the nucleotide level using BLASTN [39.40] for comparison with other Spiroplasma genomes and at the protein level using BLASTP $[39,40]$ for comparison with mycoplasma and phytoplasma genomes.

\section{Additional files}

Additional file 1: Table S1. Lists of shared and unique homologous gene clusters among S. melliferum IPMB4A (Sml), S. melliferum KC3 (SmK), and S. citri (SC). The genes in each worksheet were sorted by (1) COG categories, (2) Genome ID, (3) gene name, and (4) gene description.

Additional file 2: Table S2. Lists of shared and unique homologous gene clusters among S. melliferum IPMB4A (Sml), Mycoplasma genitalium G37 (Mg), and 'Candidatus Phytoplasma asteris' OY-M ( $\mathrm{PaO})$. The genes in each worksheet were sorted by (1) COG categories, (2) Genome ID, (3) gene name, and (4) gene description.

\section{Competing interests}

The authors declare that they have no competing interests.

\section{Authors' contributions}

WSL carried out the genome assembly, annotation, and comparative analysis. LLC handled the biological materials and carried out DNA extraction, cloning, and sequencing. WCC carried out the pulsed field gel electrophoresis and participated in genome assembly and annotation. GEG carried out the serology test and participated in the writing of the manuscript. CHK conceived of the study, isolated the bacterial strain, contributed analysis tools, participated in data analysis, and draft the manuscript. All authors read and approved the final manuscript.

\section{Acknowledgements}

We thank Dr. Chan-Pin Lin (Department of Plant Pathology and Microbiology, National Taiwan University) for advice on spiroplasma strain isolation, Ms. Mei-Jane Fang (Institute of Plant and Microbial Biology, Academia Sinica) for assistance in microscopy, the DNA Analysis Core Laboratory (Institute of Plant and Microbial Biology, Academia Sinica) for providing Sanger sequencing service, and Dr. Sen-Lin Tang (Biodiversity Research Center, Academia Sinica) for assistance in performing pulsed-field gel electrophoresis. Funding for this work was provided by research grants from Academia Sinica and the National Science Council of Taiwan (NSC 101-2621-B-001-004-MY3) to CHK. The funders had no role in the design of the study, the collection and analysis of the data, the writing of the manuscript, or the decision to submit the manuscript for publication.

\section{Author details}

${ }^{1}$ Institute of Plant and Microbial Biology, Academia Sinica, Taipei, Taiwan. ${ }^{2}$ Molecular and Biological Agricultural Sciences Program, Taiwan International Graduate Program, National Chung Hsing University and Academia Sinica, Taipei, Taiwan. ${ }^{3}$ Graduate Institute of Biotechnology, National Chung Hsing University, Taichung, Taiwan. ${ }^{4}$ Department of Biological Sciences, Towson University, Towson, MD 21252, USA. ${ }^{5}$ Biotechnology Center, National Chung Hsing University, Taichung, Taiwan.

Received: 13 September 2012 Accepted: 20 December 2012 Published: 16 January 2013

\section{References}

1. Whitcomb RF: The biology of spiroplasmas. Ann. Rev. Entomol. 1981, 26:397-425

2. Gasparich GE, Whitcomb RF, Dodge D, French FE, Glass J, Williamson DL: The genus Spiroplasma and its non-helical descendants: phylogenetic classification, correlation with phenotype and roots of the Mycoplasma mycoides clade. Int J Syst Evol Microbiol 2004, 54:893-918.

3. Regassa LB, Gasparich GE: Spiroplasmas: evolutionary relationships and biodiversity. Front Biosci 2006, 11:2983-3002.

4. Gasparich GE: Spiroplasmas and phytoplasmas: microbes associated with plant hosts. Biologicals 2010, 38:193-203.

5. Anbutsu H, Fukatsu T: Spiroplasma as a model insect endosymbiont. Env. Microbiol. Rep. 2011, 3:144-153.

6. Wu M, Eisen J: A simple, fast, and accurate method of phylogenomic inference. Genome Biol 2008, 9:R151.

7. Wu D, Hugenholtz P, Mavromatis K, Pukall R, Dalin E, Ivanova NN, Kunin V, Goodwin L, Wu M, Tindall BJ, Hooper SD, Pati A, Lykidis A, Spring S, Anderson IJ, D'haeseleer P, Zemla A, Singer M, Lapidus A, Nolan M, Copeland A, Han C, Chen F, Cheng J-F, Lucas S, Kerfeld C, Lang E, Gronow S, Chain P, Bruce D, Rubin EM, Kyrpides NC, Klenk H-P, Eisen JA: A phylogeny-driven genomic encyclopaedia of Bacteria and Archaea. Nature 2009, 462:1056-1060.

8. Bai X, Zhang J, Holford IR, Hogenhout SA: Comparative genomics identifies genes shared by distantly related insect-transmitted plant pathogenic mollicutes. FEMS Microbiol Lett 2004, 235:249-258.

9. Saglio P, Lhospital M, Lafleche D, Dupont G, Bove JM, Tully JG, Freundt EA: Spiroplasma citri gen. and sp. $\mathrm{n}$.: a mycoplasma-like organism associated with "Stubborn" disease of citrus. Int J Syst Bacteriol 1973, 23:191-204.

10. Whitcomb RF, Chen TA, Williamson DL, Liao C, Tully JG, Bove JM, Mouches C, Rose DL, Coan ME, Clark TB: Spiroplasma kunkelii sp. nov.: characterization of the etiological agent of Corn Stunt Disease. Int J Syst Bacteriol 1986, 36:170-178.

11. Saillard C, Vignault JC, Bove JM, Raie A, Tully JG, Williamson DL, Fos A, Garnier M, Gadeau A, Carle P, Whitcomb RF: Spiroplasma phoeniceum sp. nov., a new plant-pathogenic species from Syria. Int J Syst Bacterio/ 1987, 37:106-115. 
12. Nunan LM, Pantoja CR, Salazar M, Aranguren F, Lightner DV: Characterization and molecular methods for detection of a novel spiroplasma pathogenic to Penaeus vannamei. Dis. Aquat. Org. 2004, 62:255-264.

13. Nunan LM, Lightner DV, Oduori MA, Gasparich GE: Spiroplasma penaei sp. nov., associated with mortalities in Penaeus vannamei, Pacific white shrimp. Int J Syst Evol Microbiol 2005, 55:2317-2322.

14. Wang W, Gu W, Gasparich GE, Bi K, Ou J, Meng Q, Liang T, Feng Q, Zhang J, Zhang Y: Spiroplasma eriocheiris sp. nov., associated with mortality in the Chinese mitten crab, Eriocheir sinensis. Int J Syst Evol Microbiol 2011 , 61:703-708.

15. Clark TB, Whitcomb RF, Tully JG, Mouches C, Saillard C, Bove JM, Wroblewski H, Carle P, Rose DL, Henegar RB, Williamson DL: Spiroplasma melliferum, a new species from the honeybee (Apis mellifera). Int I Syst Bacteriol 1985, 35:296-308.

16. Bai $X$, Hogenhout SA: A genome sequence survey of the mollicute corn stunt spiroplasma Spiroplasma kunkelii. FEMS Microbiol Lett 2002, 210:7-17.

17. Zhao Y, Hammond RW, Jomantiene R, Dally EL, Lee I-M, Jia H, Wu H, Lin S, Zhang P, Kenton S, Najar FZ, Hua A, Roe BA, Fletcher J, Davis RE: Gene content and organization of an 85-kb DNA segment from the genome of the phytopathogenic mollicute Spiroplasma kunkelii. Mol Genet Genomics 2003, 269:592-602.

18. Carle P, Saillard C, Carrere N, Carrere S, Duret S, Eveillard S, Gaurivaud P, Gourgues G, Gouzy J, Salar P, Verdin E, Breton M, Blanchard A, Laigret F, Bove J-M, Renaudin J, Foissac X: Partial chromosome sequence of Spiroplasma citri reveals extensive viral invasion and important gene decay. Appl Environ Microbiol 2010, 76:3420-3426.

19. Alexeev D, Kostrjukova E, Aliper A, Popenko A, Bazaleev N, Tyaht A, Selezneva O, Akopian T, Prichodko E, Kondratov I, Chukin M, Demina I, Galyamina M, Kamashev D, Vanyushkina A, Ladygina V, Levitskii S, Lazarev V, Govorun V: Application of Spiroplasma melliferum proteogenomic profiling for the discovery of virulence factors and pathogenicity mechanisms in host-associated spiroplasmas. J Proteome Res 2012 11:224-236.

20. Saillard C, Carle P, Duret-Nurbel S, Henri R, Killiny N, Carrere S, Gouzy J, Bove J-M, Renaudin J, Foissac X: The abundant extrachromosomal DNA content of the Spiroplasma citri GII3-3X genome. BMC Genomics 2008, 9:195.

21. Kuo $\mathrm{CH}$, Ochman $\mathrm{H}$ : Inferring clocks when lacking rocks: the variable rates of molecular evolution in bacteria. Biol Direct 2009, 4:35.

22. Ye F, Laigret F, Bove JM: A physical and genomic map of the prokaryote Spiroplasma melliferum and its comparison with the Spiroplasma citri map. Comptes rendus de l'Académie des sciences. Série 3, Sciences de la vie 1994, 317:392-398

23. Marais A, Bove JM, Renaudin J: Characterization of the recA gene regions of Spiroplasma citri and Spiroplasma melliferum. J Bacteriol 1996, 178:7003-7009.

24. Miller RV, Kokjohn TA: General microbiology of recA: environmental and evolutionary significance. Annu Rev Microbiol 1990, 44:365-394.

25. Roca Al, Cox MM, Brenner SL: The RecA protein: structure and function. Crit Rev Biochem Mol Biol 1990, 25:415-456.

26. Brussow H, Canchaya C, Hardt W-D: Phages and the evolution of bacterial pathogens: from genomic rearrangements to lysogenic conversion. Microbiol Mol Biol Rev 2004, 68:560-602

27. Blatt J, Roces F: Haemolymph sugar levels in foraging honeybees (Apis mellifera carnica): dependence on metabolic rate and in vivo measurement of maximal rates of trehalose synthesis. J Exp Biol 2001, 204:2709-2716.

28. Gaurivaud P, Danet J-L, Laigret F, Garnier M, Bove JM: Fructose utilization and phytopathogenicity of Spiroplasma citri. Mol Plant Microbe Interact 2000, 13:1145-1155.

29. Andre A, Maucourt M, Moing A, Rolin D, Renaudin J: Sugar import and phytopathogenicity of Spiroplasma citri: glucose and fructose play distinct roles. Mol Plant Microbe Interact 2005, 18:33-42.

30. Sasaki Y, Ishikawa J, Yamashita A, Oshima K, Kenri T, Furuya K, Yoshino C, Horino A, Shiba T, Sasaki T, Hattori M: The complete genomic sequence of Mycoplasma penetrans, an intracellular bacterial pathogen in humans. Nucl. Acids Res. 2002, 30:5293-5300.

31. Killiny N, Castroviejo M, Saillard C: Spiroplasma citri spiralin acts in vitro as a lectin binding to glycoproteins from its insect vector Circulifer haematoceps. Phytopathology 2005, 95:541-548.
32. Bai X, Zhang J, Ewing A, Miller SA, Jancso Radek A, Shevchenko DV, Tsukerman K, Walunas T, Lapidus A, Campbell JW, Hogenhout SA: Living with genome instability: the adaptation of phytoplasmas to diverse environments of their insect and plant hosts. J Bacteriol 2006, 188:3682-3696.

33. Oshima K, Kakizawa S, Nishigawa H, Jung H-Y, Wei W, Suzuki S, Arashida R, Nakata D, Miyata S, Ugaki M, Namba S: Reductive evolution suggested from the complete genome sequence of a plant-pathogenic phytoplasma. Nat Genet 2004, 36:27-29.

34. Chen LL, Chung WC, Lin CP, Kuo CH: Comparative analysis of gene content evolution in phytoplasmas and mycoplasmas. PLoS One 2012, 7:e34407.

35. Lin CP: Spiroplasmas isolated from honey bee (Apis mellifera L.) in Taiwan:: MS thesis. National Taiwan University, Department of Plant Pathology; 1980

36. Moulder RW, French FE, Chang CJ: Simplified media for spiroplasmas associated with tabanid flies. Can J Microbiol 2002, 48:1-6.

37. Padovan AC, Firrao G, Schneider B, Gibb KS: Chromosome mapping of the sweet potato little leaf phytoplasma reveals genome heterogeneity within the phytoplasmas. Microbiology 2000, 146:893-902.

38. Dally EL, Barros TS, Zhao Y, Lin S, Roe BA, Davis RE: Physical and genetic map of the Spiroplasma kunkelii CR2-3x chromosome. Can J Microbiol 2006, 52:857-867.

39. Altschul SF, Gish W, Miller W, Myers EW, Lipman DJ: Basic local alignment search tool. J Mol Biol 1990, 215:403-410.

40. Camacho C, Coulouris G, Avagyan V, Ma N, Papadopoulos J, Bealer K, Madden T: BLAST+: architecture and applications. BMC Bioinformatics 2009, 10:421

41. Benson DA, Karsch-Mizrachi I, Clark K, Lipman DJ, Ostell J, Sayers EW: GenBank. Nucl. Acids Res. 2012, 40:D48-D53.

42. Edgar RC: MUSCLE: multiple sequence alignment with high accuracy and high throughput. Nucl. Acids Res. 2004, 32:1792-1797.

43. Guindon S, Gascuel O: A simple, fast, and accurate algorithm to estimate large phylogenies by maximum likelihood. Syst Biol 2003, 52:696-704.

44. Felsenstein J: PHYLIP - Phylogeny Inference Package (Version 3.2). Cladistics 1989, 5:164-166.

45. Williamson D, Whitcomb R, Tully J: The spiroplasma deformation test, a new serological method. Curr Microbiol 1978, 1:203-207.

46. Chitsaz H, Yee-Greenbaum JL, Tesler G, Lombardo M-J, Dupont CL, Badger JH, Novotny M, Rusch DB, Fraser LJ, Gormley NA, Schulz-Trieglaff O, Smith GP, Evers DJ, Pevzner PA, Lasken RS: Efficient de novo assembly of single-cell bacterial genomes from short-read data sets. Nat. Biotech. 2011, 29:915-921.

47. Li H, Durbin R: Fast and accurate short read alignment with BurrowsWheeler transform. Bioinformatics 2009, 25:1754-1760.

48. Robinson JT, Thorvaldsdottir H, Winckler W, Guttman M, Lander ES, Getz G, Mesirov JP: Integrative genomics viewer. Nat. Biotech. 2011, 29:24-26.

49. Li H, Handsaker B, Wysoker A, Fennell T, Ruan J, Homer N, Marth G, Abecasis G, Durbin R, 1000 Genome Project Data Processing Subgroup: The Sequence Alignment/Map format and SAMtools. Bioinformatics 2009, 25:2078-2079.

50. Green P: Phrap:: http://www.phrap.org/.

51. Lagesen $\mathrm{K}$, Hallin $\mathrm{P}$, Rodland EA, Staerfeldt $\mathrm{H}-\mathrm{H}$, Rognes $\mathrm{T}$, Ussery DW: RNAmmer: consistent and rapid annotation of ribosomal RNA genes. Nucl. Acids Res. 2007, 35:3100-3108.

52. Lowe T, Eddy S: tRNAscan-SE: a program for improved detection of transfer RNA genes in genomic sequence. Nucl. Acids Res. 1997, 25:955-964.

53. Hyatt D, Chen G-L, LoCascio P, Land M, Larimer F, Hauser L: Prodigal: prokaryotic gene recognition and translation initiation site identification. BMC Bioinformatics 2010, 11:119.

54. Li L, Stoeckert CJ, Roos DS: OrthoMCL: Identification of ortholog groups for eukaryotic genomes. Genome Res 2003, 13:2178-2189.

55. Moriya Y, Itoh M, Okuda S, Yoshizawa AC, Kanehisa M: KAAS: an automatic genome annotation and pathway reconstruction server. Nucl. Acids Res. 2007, 35:W182-W185.

56. Kanehisa M, Goto S: KEGG: Kyoto encyclopedia of genes and genomes. Nucl. Acids Res. 2000, 28:27-30.

57. Kanehisa M, Goto S, Furumichi M, Tanabe M, Hirakawa M: KEGG for representation and analysis of molecular networks involving diseases and drugs. Nucl. Acids Res. 2010, 38:D355-D360.

58. Tatusov RL, Koonin EV, Lipman DJ: A genomic perspective on protein families. Science 1997, 278:631-637. 
59. Tatusov R, Fedorova N, Jackson J, Jacobs A, Kiryutin B, Koonin E, Krylov D, Mazumder R, Mekhedov S, Nikolskaya A, Rao BS, Smirnov S, Sverdlov A, Vasudevan S, Wolf Y, Yin J, Natale D: The COG database: an updated version includes eukaryotes. BMC Bioinformatics 2003, 4:41.

60. Darling ACE, Mau B, Blattner FR, Perna NT: Mauve: multiple alignment of conserved genomic sequence with rearrangements. Genome Res 2004, 14:1394-1403.

doi:10.1186/1471-2164-14-22

Cite this article as: Lo et al:: Comparative genome analysis of

Spiroplasma melliferum IPMB4A, a honeybee-associated bacterium. BMC Genomics 2013 14:22.

\section{Submit your next manuscript to BioMed Central and take full advantage of:}

- Convenient online submission

- Thorough peer review

- No space constraints or color figure charges

- Immediate publication on acceptance

- Inclusion in PubMed, CAS, Scopus and Google Scholar

- Research which is freely available for redistribution 